\title{
Efeito de níveis de fósforo não-fítico e de fitase sobre o fêmur de frangos de corte
}

\author{
Luana Martins Schaly ${ }^{1}$ \\ Bruno Nunes Gonçalves ${ }^{2}$ \\ Maria Cristina de Oliveira ${ }^{1 *}$ \\ Antônio Carlos Laurentiz ${ }^{3}$ \\ Otto Mack Junqueira ${ }^{4}$ \\ 'Faculdade de Medicina Veterinária, Universidade de Rio Verde, Rio Verde - GO, Brasil \\ ${ }^{2}$ Faculdade de Zootecnia, Universidade de Rio Verde \\ ${ }^{3}$ Faculdade de Engenharia de Ilha Solteira, UNESP, Ilha Solteira - SP, Brasil \\ ${ }^{4}$ FCAV, UNESP, Jaboticabal - SP, Brasil \\ *Autor para correspondência \\ cristina@fesurv.br
}

Submetido em 26/03/2008

Aceito para publicação em 04/11/2008

\section{Resumo}

Esse experimento foi conduzido para avaliar o efeito de níveis de fósforo não-fítico (FNF) e de fitase sobre peso, morfometria e índice peso/comprimento (IPC) de fêmures de frangos aos 21 e 42 dias de idade. Foram utilizados 1200 pintos de corte alojados em delineamento inteiramente casualizado e arranjo fatorial 4 x 3 (níveis de FNF x níveis de fitase), com quatro repetições. Os níveis de FNF, em cada fase, foram de 0,45; 0,$37 ; 0,29$ e $0,21 \%$ na fase inicial; 0,$41 ; 0,33 ; 0,25$ e $0,17 \%$ na fase de crescimento e de 0,$37 ; 0,29 ; 0,21$ e $0,13 \%$ na fase final. Os níveis de fitase utilizados foram 0,500 e 1000U/kg de dieta. Aos 21 e 42 dias de idade, 48 aves foram sacrificadas para a retirada dos fêmures. Aos 21 dias, não houve efeito $(P>0,05)$ da interação FNF x fitase sobre parâmetros ósseos, porém a redução do FNF dietético diminuiu $(\mathrm{P}<0,05)$ o peso, o comprimento e o IPC dos fêmures e a inclusão de 500U/kg de fitase melhorou $(\mathrm{P}<0,05)$ o peso e o IPC dos ossos. Aos 42 dias de criaçãi, a interação FNF x fitase foi significativa $(\mathrm{P}<0,05)$ para peso e comprimento dos fêmures, em que aves que ingeriram dietas sem fitase tiveram fêmures mais leves e mais curtos quando o menor nível de FNF foi avaliado, porém, a inclusão de $500 \mathrm{ou} 1000 \mathrm{U} / \mathrm{kg}$ de fitase tornou o peso e o comprimento similares aos do tratamento com níveis recomendados de FNF e o menor nível de FNF avaliado, promoveu redução $(\mathrm{P}<0,05)$ no diâmetro e no IPC dos fêmures. Concluiu-se que dietas com 0,29, 0,25 e 0,21\% de FNF, suplementadas com $500 \mathrm{U} / \mathrm{kg}$ de fitase, podem ser usadas sem prejuízos para a qualidade do fêmur dos frangos de corte de um a 42 dias de idade.

Unitermos: enzimas, qualidade óssea, deficiência de fósforo

\section{Abstract}

Effect of nonphytate phosphorus and phytase levels on broiler femur. This experiment was carried out to evaluate the effect of nonphytate phosphorus (NPP) and phytase levels on the weight, morphometry and 
weight/length index (WLI) of broiler femurs at 21 and 42 days of age. One thousand, two hundred chicks were allocated in a completely randomized design and $4 \times 3$ factorial arrangement (NPP x phytase levels), with four replicates. The NPP levels, at each phase were of $0.45,0.37,0.29$ and $0.21 \%$ in the initial phase, $0.41,0.33,0.25$ and $0.17 \%$ in the growth phase, and of $0.37,0.29,0.21$ and $0.13 \%$ in the final phase. The phytase levels used were 0,500 and $1000 \mathrm{U} / \mathrm{kg}$ of diet. At 21 and 42 days of age, 48 birds were sacrificed for femur collection. At 21 days, there was no effect $(\mathrm{P}>0.05)$ of NPP $\mathrm{x}$ phytase interaction on bone parameters, but the NPP reduction decreased $(\mathrm{P}<0.05)$ the weight, length and WLI of the femurs, and the inclusion of $500 \mathrm{U} / \mathrm{kg}$ of phytase improved $(\mathrm{P}<0.05)$ the weight and WLI of the bones. At 42 days of age, NPP x phytase interaction was significant $(\mathrm{P}$ $<0.05)$ for the weight and length, and birds fed diets with no phytase had femurs that were lighter and shorter when the lowest NPP levels were evaluated. However, the inclusion of 500 or $1000 \mathrm{U} / \mathrm{kg}$ of phytase produced weights and lengths similar to those produced by treatment with recommended NPP levels, and the lower NPP levels evaluated caused a reduction $(\mathrm{P}<0.05)$ in the diameter and WLI of femurs. It was concluded that diets with $0.29,0.25$ or $0.21 \%$ of NPP, with $500 \mathrm{U} / \mathrm{kg}$ of phytase, could be used with no negative effect on the femur quality in broilers from one to 42 days of age.

Key words: bone quality, enzymes, phosphorus deficiency

\section{Introdução}

O fósforo $(\mathrm{P})$ é o segundo mineral mais abundante no corpo sendo que a maior parte é encontrada nos ossos. Além disso, é essencial para o crescimento e desenvolvimento dos ossos nos animais. A deficiência de $P$ pode resultar em deformidades ósseas, as quais são responsáveis pela desclassificação da carcaça durante o processamento (Brenes et al., 2003). Pernas fracas estão associadas à menor ingestão de alimentos afetando, assim, o ganho de peso (Onyango et al., 2003).

Aproximadamente $70 \%$ do $\mathrm{P}$ dos grãos de cereais e farelos de sementes oleaginosas está na forma de fitato ( $\mathrm{P}$ fítico), sendo esta a principal fonte natural de $P$ no alimento animal (Casey e Walsh, 2004). A disponibilidade do P fítico de alimentos vegetais é reduzida em animais monogástricos devido à pouca ou nenhuma atividade de fitase no trato gastrintestinal (Vats e Banerjee, 2004). Sendo assim, fósforo inorgânico (Pi) é adicionado às dietas de monogástricos para compensar a utilização ineficiente do $P$ fítico (Jendza et al., 2006). Entretanto, deve-se considerar que o Pé o terceiro nutriente mais caro em dietas avícolas, após a energia e proteína (Biehl et al., 1998), além de ser um contaminante ambiental. A inclusão de fitase nas dietas poderia ser útil na redução de sua excreção diminuindo, assim, o poder poluente da cama de frango (Silva et al., 2008).

As fitases hidrolisam o ácido fítico e seus sais, produzindo inositol, inositol monofosfato e P inorgânico (Casey e Walsh, 2004) e a suplementação das dietas com estas enzimas pode melhorar o crescimento ósseo (Ahmad et al., 2000).
A adição de fitase a dietas de frangos pode ser economicamente viável por melhorar a conversão alimentar (Costa et al., 2007) e aumentar o peso corporal das aves (Tsokova et al., 2004) proporcionalmente à quantidade de fitase adicionada.

Vários métodos invasivos (teor de matéria mineral, resistência à quebra, por exemplo) e não-invasivos (ultrasom) existem para determinar a mineralização óssea em frangos (Onyango et al., 2003). Entretanto, Seedor et al. (1991) utilizaram o índice peso/comprimento (IPC) para estimar a mineralização óssea e encontraram uma alta correlação entre IPC e densidade óssea.

Sendo assim, esse experimento foi conduzido para avaliar o efeito de níveis dietéticos de fósforo não-fítico (FNF) e da enzima fitase sobre o peso, a morfometria e o IPC de fêmures de frangos de corte aos 21 e 42 dias de idade.

\section{Material e Métodos}

Foram utilizados 1200 pintos de corte machos, da linhagem Cobb, com um dia de idade, alojados em galpão de alvenaria dividido em boxes de 2,8 $\mathrm{m}$ x 1,6m (área total de $4,48 \mathrm{~m}^{2}$ ). O manejo adotado para as fases de criação seguiu as recomendações do manual da linhagem.

$\mathrm{O}$ delineamento experimental foi inteiramente casualizado em fatorial $4 \times 3$ (quatro níveis de FNF $x$ três níveis de fitase), com quatro repetições de 25 aves cada. Os níveis de fósforo não-fítico, em cada fase, foram de 0,$45 ; 0,37 ; 0,29$ e $0,21 \%$ na fase inicial ( 1 a 21 dias); 0,41 ; 0,$33 ; 0,25$ e $0,17 \%$ na fase de crescimento ( 22 a 35 dias) e 
de 0,$37 ; 0,29 ; 0,21$ e $0,13 \%$ na fase final (36 a 42 dias de idade). A redução dos níveis de FNF das dietas, nas fases inicial (I), crescimento (C) e terminação (T), foi de 18, 36 e $54 \% ; 19,38$ e $58 \% ; 21$, 43 e $64 \%$, respectivamente. Os níveis de fitase utilizados foram 0,100 e $200 \mathrm{~g} /$ tonelada, garantindo 0,500 e $1000 \mathrm{FTU} / \mathrm{kg}$ de dieta.

Os tratamentos foram os seguintes: $\mathrm{T}_{1}-0,45 \%$ (I), $0,41 \%$ (C) e $0,37 \%$ (T) FNF; $\mathrm{T}_{2}-\mathrm{T} 1+500 \mathrm{U} / \mathrm{kg}$ de fitase; $\mathrm{T}_{3}-\mathrm{T} 1+1000 \mathrm{U} / \mathrm{kg}$ de fitase; $\mathrm{T}_{4}-0,37 \%(\mathrm{I}), 0,33 \%(\mathrm{C})$ e $0,29 \%(\mathrm{~T}) \mathrm{FNF} ; \mathrm{T}_{5}-\mathrm{T} 4+500 \mathrm{U} / \mathrm{kg}$ de fitase; $\mathrm{T}_{6}-\mathrm{T} 4+$ $1000 \mathrm{U} / \mathrm{kg}$ de fitase; $\mathrm{T}_{7}-0,29 \%(\mathrm{I}), 0,25 \%(\mathrm{C})$ e $0,21 \%(\mathrm{~T})$ FNF; $\mathrm{T}_{8}-\mathrm{T} 7+500 \mathrm{U} / \mathrm{kg}$ de fitase; $\mathrm{T}_{9}-\mathrm{T} 7+1000 \mathrm{U} / \mathrm{kg}$ de fitase; $\mathrm{T}_{10}-0,21 \%(\mathrm{I}), 0,17 \%(\mathrm{C})$ e $0,13 \%(\mathrm{~T}) \mathrm{FNF} ; \mathrm{T}_{11}-\mathrm{T} 10$ $+500 \mathrm{U} / \mathrm{kg}$ de fitase e $\mathrm{T}_{12}-\mathrm{T} 10+1000 \mathrm{U} / \mathrm{kg}$ de fitase.

A enzima fitase utilizada foi a Natuphos $5.000^{\circledR}$, obtida por intermédio da fermentação por fungos Aspergillus niger, contendo atividade inicial mínima, declarada pelo fabricante, de $5.000 \mathrm{FTU} / \mathrm{g}$ do produto. As dietas experimentais fareladas eram isonutritivas e foram formuladas de acordo as recomendações nutricionais e a composição dos ingredientes de Rostagno et al. (2005), exceto para os valores de fósforo não-fítico.
Quatro aves de cada tratamento foram sacrificadas, aos 21 e aos 42 dias de idade, por deslocamento cervical e delas foram retirados os fêmures direitos. Todos os fêmures, após serem extraídos, foram limpos dos tecidos aderentes. Os fêmures foram utilizados para obtenção do peso e para a morfometria (comprimento e diâmetro da porção média do osso) obtida com paquímetro manual. $\mathrm{O}$ índice peso/comprimento foi obtido dividindo-se o peso, em mg, pelo comprimento, em mm (Kocabagli, 2001).

Os resultados obtidos foram submetidos à análise de variância por meio do programa SAEG (UFV, 2001) e as médias foram comparadas pelo teste de Tukey a 5\% de probabilidade.

\section{Resultados e Discussão}

Não houve efeito $(\mathrm{P}>0,05)$ da interação FNF $\mathrm{x}$ fitase sobre parâmetros ósseos aos 21 dias de idade (Tabela 1). A redução dos níveis de FNF dietético para menos que 0,37 e $0,29 \%$, resultou em menores $(\mathrm{P}<0,05)$ peso e IPC e comprimento dos fêmures, respectivamente.

TABELA 1: Peso, morfometria e índice peso/comprimento de fêmures de frangos, com 21 dias de idade, alimentados com dietas com níveis de fósforo não-fítico e de fitase.

\begin{tabular}{|c|c|c|c|c|c|c|c|}
\hline \multirow[b]{2}{*}{ Parâmetro } & \multirow[b]{2}{*}{$\begin{array}{l}\text { Fitase } \\
\text { (U/kg) }\end{array}$} & \multicolumn{4}{|c|}{ Nível de fósforo não-fítico (\%) } & \multirow[b]{2}{*}{ Média } & \multirow{2}{*}{$\begin{array}{l}\mathrm{CV} \\
(\%)\end{array}$} \\
\hline & & 0,45 & 0,37 & 0,29 & 0,21 & & \\
\hline \multirow[t]{4}{*}{ Peso (g) } & 0 & 4,69 & 4,16 & 3,67 & 3,14 & $3,91 \mathrm{~b}$ & \\
\hline & 500 & 4,99 & 4,56 & 4,26 & 3,93 & $4,43 \mathrm{a}$ & \\
\hline & 1000 & 4,91 & 4,94 & 4,48 & 3,98 & $4,57 \mathrm{a}$ & \\
\hline & Média & $4,86 \mathrm{a}$ & $4,55 \mathrm{ab}$ & $4,14 b c$ & $3,68 \mathrm{c}$ & & 12,47 \\
\hline \multirow[t]{4}{*}{ Diâmetro (mm) } & 0 & 6,75 & 6,50 & 6,12 & 6,50 & 6,46 & \\
\hline & 500 & 6,37 & 6,75 & 6,00 & 6,50 & 6,40 & \\
\hline & 1000 & 6,50 & 6,25 & 6,37 & 6,12 & 6,31 & \\
\hline & Média & 6,54 & 6,50 & 6,16 & 6,37 & & 9,02 \\
\hline \multirow[t]{4}{*}{ Comprimento (mm) } & 0 & 55,00 & 53,25 & 52,00 & 45,17 & 51,35 & \\
\hline & 500 & 55,75 & 53,62 & 53,25 & 50,62 & 53,31 & \\
\hline & 1000 & 55,00 & 53,12 & 55,00 & 49,25 & 53,09 & \\
\hline & Média & $55,25 \mathrm{a}$ & $53,33 \mathrm{a}$ & $53,41 \mathrm{a}$ & $48,35 b$ & & 5,07 \\
\hline \multirow[t]{4}{*}{ IPC (mg/mm) } & 0 & 85,27 & 78,12 & 70,57 & 69,51 & $58,37 \mathrm{~b}$ & \\
\hline & 500 & 89,51 & 85,04 & 80,00 & 77,64 & $83,04 \mathrm{a}$ & \\
\hline & 1000 & 89,27 & 92,99 & 81,45 & 80,81 & $86,13 a$ & \\
\hline & Média & $88,01 \mathrm{a}$ & $85,38 \mathrm{a}$ & $77,34 b$ & $75,98 b$ & & 9,90 \\
\hline
\end{tabular}

$\mathrm{CV}=$ coeficiente de variação. Médias seguidas de letras diferentes diferem entre si pelo teste de Tukey. 
O nível de $0,37 \%$ de FNF parece ser suficiente para manter o peso, o comprimento e o IPC normais durante a fase inicial de criação. Nessa fase, os minerais são utilizados não só para o crescimento do tecido ósseo, mas também de outros órgãos e tecidos. Entretanto, a exigência de $\mathrm{P}$ foi atendida com um nível abaixo do remendado na literatura, de $0,45 \%$.

A inclusão de 500U/kg de fitase melhorou $(\mathrm{P}<0,05)$ o peso e o IPC dos ossos. Para que o osso cresça, é necessária a presença de minerais em quantidades adequadas. Como a fitase é uma enzima que hidrolisa a molécula de fitato liberando o P fítico, é possível que tenha ocorrido aumento na absorção (Tamin et al., 2004) e utilização de P pelos ossos. Como houve aumento no peso, mas não no comprimento, o IPC foi maior e quanto maior o IPC mais denso é o osso.

A interação FNF $\mathrm{x}$ fitase foi significativa $(\mathrm{P}<0,05)$ para peso e comprimento dos fêmures aos 42 dias de criação (Tabela 2). As aves que ingeriram dietas sem fitase tiveram fêmures mais leves e mais curtos quando o menor nível de FNF foi avaliado, porém, a inclusão de 500 ou $1000 \mathrm{U} / \mathrm{kg}$ de fitase tornou o peso e o comprimento similares aos do tratamento com níveis recomendados de FNF. O menor nível de FNF avaliado promoveu redução $(\mathrm{P}<0,05)$ no diâmetro e no IPC dos fêmures.

Nas fases de crescimento e final, os órgãos e tecidos já estão formados e o metabolismo da ave é dirigido principalmente para a deposição protéica no músculo, que é maior comparada com a fase inicial. Portanto, a exigência de P nessa fase é menor o que permitiu que níveis ainda menores de FNF pudessem ser utilizados, desde que a dieta fosse suplementada com fitase, sem que houvesse prejuízo no peso e comprimento dos fêmures. Entretanto, independente da inclusão de fitase, o menor nível de FNF avaliado não foi suficiente para manter o diâmetro e o IPC dos fêmures. Nessa fase, o peso da ave é maior e, de acordo com Wasserman et al. (1996), o aumento da massa muscular é acompanhado do aumento da massa óssea, o que justificaria o maior diâmetro e IPC.

A tíbia é o osso mais comumente utilizado para determinação de exigências de minerais, pois é o principal osso de sustentação do corpo da ave. Sendo assim, as informações na literatura a respeito da responsividade do fêmur a alterações nos minerais da dieta são escassas.

TABELA 2: Peso, morfometria e índice peso/comprimento de fêmures de frangos, com 42 dias de idade, alimentados com dietas com níveis de fósforo não-fítico e de fitase.

\begin{tabular}{|c|c|c|c|c|c|c|c|}
\hline \multirow[b]{2}{*}{ Parâmetro } & \multirow[b]{2}{*}{$\begin{array}{l}\text { Fitase } \\
(\mathrm{U} / \mathrm{kg})\end{array}$} & \multicolumn{4}{|c|}{ Nível de fósforo não-fítico (\%) } & \multirow[b]{2}{*}{ Média } & \multirow[b]{2}{*}{$\begin{array}{l}\text { CV } \\
(\%)\end{array}$} \\
\hline & & $\begin{array}{c}0,45-0,41- \\
0,37\end{array}$ & $\begin{array}{c}0,37-0,33- \\
0,29\end{array}$ & $\begin{array}{c}0,29-0,25- \\
0,21\end{array}$ & $\begin{array}{c}0,21-0,17- \\
0,13\end{array}$ & & \\
\hline \multirow[t]{4}{*}{ Peso (g) } & 0 & $11,91 \mathrm{Aa}$ & $11,74 \mathrm{Aa}$ & $12,66 \mathrm{Aa}$ & $6,94 \mathrm{Bb}$ & 10,81 & \\
\hline & 500 & $11,64 \mathrm{Aa}$ & $11,27 \mathrm{Aa}$ & $10,45 \mathrm{Aa}$ & $9,68 \mathrm{Aa}$ & 10,76 & \\
\hline & 1000 & $12,29 \mathrm{Aa}$ & $12,08 \mathrm{Aa}$ & $10,39 \mathrm{Aa}$ & $9,43 \mathrm{Aa}$ & 11,04 & \\
\hline & Média & 11,95 & 11,69 & 11,16 & 8,68 & & 12,88 \\
\hline \multirow{4}{*}{$\begin{array}{l}\text { Diâmetro } \\
(\mathrm{mm})\end{array}$} & 0 & 9,50 & 9,12 & 8,62 & 7,75 & 8,75 & \\
\hline & 500 & 9,37 & 8,62 & 8,75 & 8,50 & 8,81 & \\
\hline & 1000 & 9,75 & 9,12 & 9,75 & 8,37 & 9,25 & \\
\hline & Média & $9,54 a$ & $8,95 \mathrm{a}$ & $9,04 \mathrm{a}$ & $8,20 b$ & & 14,48 \\
\hline \multirow{4}{*}{$\begin{array}{l}\text { Comprimento } \\
(\mathrm{mm})\end{array}$} & 0 & $75,37 \mathrm{Aa}$ & $74,87 \mathrm{Aa}$ & $75,62 \mathrm{Aa}$ & $62,50 \mathrm{Bb}$ & 72,09 & \\
\hline & 500 & $73,25 \mathrm{Aa}$ & $74,62 \mathrm{Aa}$ & $72,62 \mathrm{Aa}$ & $71,87 \mathrm{Aa}$ & 73,09 & \\
\hline & 1000 & $75,87 \mathrm{Aa}$ & $76,25 \mathrm{Aa}$ & $71,87 \mathrm{Aa}$ & $71,25 \mathrm{Aa}$ & 73,81 & \\
\hline & Média & 74,83 & 75,25 & 73,37 & 68,54 & & 4,58 \\
\hline IPC & 0 & 157,43 & 156,84 & 166,99 & 110,71 & 147,99 & \\
\hline \multirow[t]{3}{*}{$(\mathrm{mg} / \mathrm{mm})$} & 500 & 158,92 & 150,41 & 143,82 & 134,73 & 146,97 & \\
\hline & 1000 & 162,13 & 158,38 & 144,49 & 132,14 & 149,28 & \\
\hline & Média & $159,49 a$ & $155,21 \mathrm{a}$ & $151,77 \mathrm{a}$ & $125,86 \mathrm{~b}$ & & 10,50 \\
\hline
\end{tabular}

$\mathrm{CV}=$ coeficiente de variação. Médias seguidas de letras diferentes minúsculas nas linhas e maiúsculas nas colunas, diferem entre si pelo teste de Tukey. 
Resultados semelhantes foram relatados por Viveros et al. (2002), que estudaram dietas com níveis reduzidos de FNF $(0,45$ a $0,24 \%)$ suplementadas ou não com 500U/ $\mathrm{kg}$ de fitase e notaram que houve redução no peso da tíbia com os menores níveis de FNF, porém, a suplementação de dietas com fitase melhorou o peso dos ossos. Abdellatif e Kamal (2003) relataram que a inclusão de $750 \mathrm{U} / \mathrm{kg}$ de fitase em dietas com $0,4 \%$ de FNF melhorou o peso e o comprimento de tíbias e Jamroz et al. (2007) verificaram que menores níveis de $\mathrm{P}$ dietéticos resultam em menores pesos de tíbias em frangos.

Já Orban et al. (1999) e Kocabagli (2001) relataram que a inclusão de 0 a $1500 \mathrm{U} / \mathrm{kg}$ de fitase em dietas com níveis de $\mathrm{P}$ reduzidos para patos e frangos com 42 dias de idade, não afetou o peso, comprimento e diâmetro de tíbias.

Com base no exposto, conclui-se que é possível a utilização de dietas com 0,29, 0,25 e 0,21\% de FNF nas fases inicial, crescimento e terminação, respectivamente, suplementadas com $500 \mathrm{U} / \mathrm{kg}$ de fitase, sem prejuízos para a qualidade do fêmur dos frangos de corte de um a 42 dias de idade.

\section{Referências}

Abdellatif, H. A.; Kamal, A. M. 2003. Effect of phytase supplementation on the performance of broilers grown to market weights. Veterinary Medicine Journal of Giza, 51 (3): 343-354.

Ahmad, T.; Rasool, S.; Sarwar, M.; Haq, A.; Hasan, Z. 2000. Effect of microbial phytase produced from a fungus Aspergillus niger on bioavailability of phosphorus and calcium in broiler chickens. Animal Feed Science and Technology, 83 (2): 103-114.

Biehl, R. R.; Baker, D. H.; de Luca, H. F. 1998. Activity of various vitamin $\mathrm{D}_{3}$ analogs for improving phosphorus utilization in chicks receiving diets adequate in vitamin $\mathrm{D}_{3}$. British Poultry Science, 39 (3): 408-412.

Brenes, A.; Viveros, A.; Arija, I.; Centeno, C.; Pizarro, M.; Bravo, C. 2003. The effect of citric acid and microbial phytase on mineral utilization in broiler chicks. Animal Feed Science and Technology, 110 (1-4): 201-219.

Casey, A.; Walsh, G. 2004. Identification and characterization of a phytase of potential commercial interest. Journal of Biotechnology, 110 (3): 313-322.

Costa, F. G. P.; Brandão, P. A.; Brandão, J. S.; Silva, J. H. V. 2007. Efeito da enzima fitase nas rações de frangos de corte, durante as fases pré-inicial e inicial. Ciência e Agrotecnologia, 31 (3): 865 870.

Jamroz, D.; Wertelecki, T.; Zylka, R. 2007. The retention of mineral substances, quality and chemical composition of bones of chickens fed diets containing different calcium and phosphorus levels. Eletronic Journal of Polish Agricultural Universities, 10 (3): Disponível em <http://www.ejpau.media.pl/volume10/issue3/art-04. html>. Acesso em 08 de fevereiro de 2008.

Jendza, J. A.; Dilger, R. N.; Sands, J. S.; Adeola, O. 2006. Efficacy and equivalency of an Escherichia coli-derived phytase for replacing inorganic phosphorus in the diets of broiler chickens and young pig. Journal of Animal Science, 84 (12): 3364-3374.

Kocabagli, N. 2001. The effect of dietary phytase supplementation at different levels on tibial bone characteristics and strength in broilers. Turkish Journal of Veterinary and Animal Science, 25 (5): 797-802

Onyango, E. M.; Hester, P. Y.; Stroshine, R.; Adeola, O. 2003. Bone densitometry as an indicator of percentage tibia ash in broiler chicks fed varying dietary calcium and phosphorus levels. Poultry Science, 82 (11): 1787-1791.

Orban, J. I.; Adeola, O.; Stroshine, R. 1999. Microbial phytase in finisher diets of White Pekin ducks: effect on growth performance, plasma phosphorus concentration, and leg bone characteristics. Poultry Science, 78 (3): 366-377.

Rostagno, H. S.; Albino, L. F. T.; Donzele, J. L.; Gomes, P. C.; Oliveira, R. F.; Lopes, D. C.; Ferreira, A. S.; Barreto, S. T 2005. Tabelas brasileiras para aves e suínos. Composição de alimentos $\mathrm{e}$ exigências nutricionais. $2^{\mathrm{a}} \mathrm{ed}$. UFV, Viçosa, Brasil, 186pp.

Seedor, J. G.; Quarruccio, H. A.; Thompson, D. D. 1991. The bisphosphonate alendronate (MK-217) inhibits bone loss due to ovariectomy in rats. Journal of Bone and Mineral Research, 6 (4): 339-346.

Silva, Y. L.; Rodrigues, P. B.; Freitas, R. T. F.; Zangeronimo, M. G.; Fialho, E. T. 2008. Níveis de proteína e fósforo em rações com fitase para frangos de corte, na fase de 14 a 21 dias de idade. 2. Valores energéticos e digestibilidade de nutrientes. Revista Brasileira de Zootecnia, 37 (3): 469-477.

Tamin, N. M.; Angel, R.; Christman, M. 2004. Influence of dietary calcium and phytase on phytase phosphorus hydrolysis in broiler chickens. Poultry Science, 83 (8): 1358-1367.

Tsokova, L.; Kanakov, D.; Stoyanchev, K. 2004. Influence of various amounts of phytase and dicalcium phosphate in the diet of growing chickens upon the weight and strength of their bones. Trakia Journal of Sciences, 3 (Suppl. 2): 71-74.

UFV - Universidade Federal de Viçosa. 2001. SAEG - Sistema de análises estatísticas e genéticas. Versão 8.0. UFV, Viçosa, Brasil, $150 \mathrm{pp}$.

Vats, P.; Banerjee, U. C. 2004. Production studies and catalytic properties of phytases (myo-inositolhexakisphosphate phosphohydrolases): an overview. Enzyme and Microbial Technology, 35 (1): 3-14.

Viveros, A.; Brenes, A.; Arija, I.; Centeno, C. 2002. Effects of microbial phytase supplementation on mineral utilization and serum enzyme activities in broiler chicks fed different levels of phosphorus. Poultry Science, 81 (8): 1172-1183.

Wasserman, R. H.; Kallfelz, F. A.; Lust, G. 1996. Ossos, articulações e líquido sinovial. In: Swenson, M. J. \& Reece, W. O. (Eds). Dukes - Fisiologia dos Animais Domésticos. $11^{\mathrm{a}}$ ed. Guanabara Koogan S.A., Rio de Janeiro, Brasil, p.499. 